\title{
Improving T-Wave Alternans Measurement Quality by Reducing Noise and Artifacts
}

\author{
W Kaiser ${ }^{1}$, M Findeis ${ }^{1}$, BJ Young ${ }^{2}$ \\ ${ }^{1}$ GE Healthcare Information Technologies, Freiburg, Germany \\ ${ }^{2}$ GE Healthcare Information Technologies, Milwaukee, WI, USA
}

\begin{abstract}
We adopted the time domain method for T-wave alternans (TWA) measurement from BD Nearing and $R L$ Verrier, Harvard Medical School, Boston, MA, USA, developed techniques for noise and artifact rejection, and made it more suitable for processing of ECG data, acquired with standard electrodes during exercise stress testing and Holter monitoring. New techniques are cubic alignment, nonlinear filtering, and intra class variability in the odd and even median beats. During the development stage 64 simulated ECGs with different amplitudes, heart rates, QT intervals, TWA levels, and different noise types and levels were used. With these ECGs the expected TWA values were known. Their agreement with the measured values was very high. In addition, we used 90 real Holter, 1416 bicycle and treadmill exercise test ECGs with annotated visible TWA episodes for development and verification of the TWA algorithm.
\end{abstract}

\section{Introduction}

Electrical alternans affecting the ST-segment and Twave has been observed among patients at increased risk for ventricular arrhythmias. Although the electrical alternans affects both the ST-segment and the T-wave, henceforward the alternans is referred to as "T-wave alternans" or TWA.

Subtle T-wave alternans on the electrocardiogram may serve as a noninvasive marker of vulnerability to ventricular tachyarrhythmias [1]. An example of this clinical phenomenon is shown in Figure 1.

The method for detection of electrical alternans in the ST-segment and T-wave, described here, was invented by Bruce D.Nearing and Richard L.Verrier, Harvard Medical School, Boston, MA, USA [2]. We adopted the method and developed additional algorithms to make it robust and tolerant to noise and artifacts.

The method directly measures the morphologic fluctuations between odd and even beats in the time domain without mathematical transformation, which allows visual confirmation of measurements by an overreader.

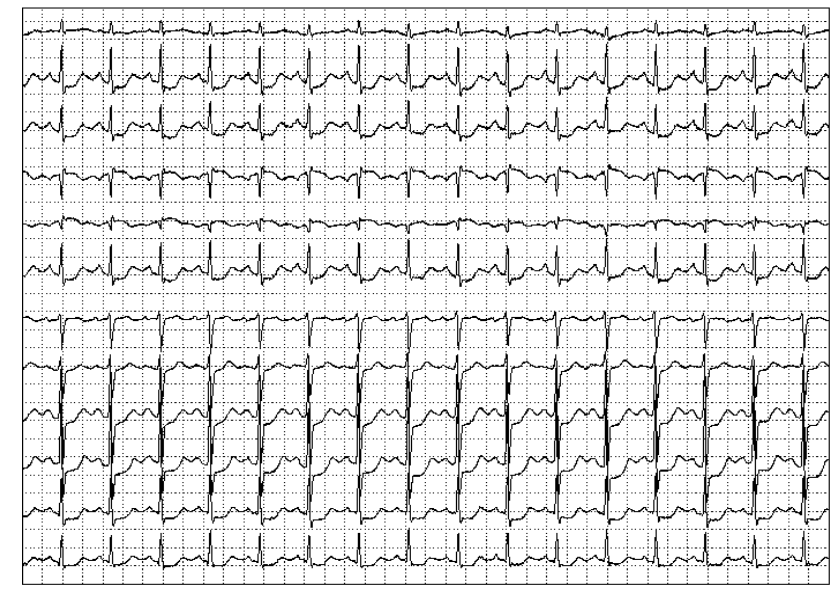

Figure 1. 12 lead ECG from a bicycle exercise test. Twave alternans is visible in the $10^{\text {th }}$ trace (lead V4). The amplitude of the ST-T segment changes from beat to beat in an alternating pattern of higher and lower ST levels.

\section{Methods}

\subsection{Data}

Data used to test and improve the method included the following:

- $\quad$ simulated ECGs with different TWA values, baseline shifts, noise, amplitudes, and heart rates (64 patients, 2 leads)

- Holter ECGs from the ESC (European Society of Cardiology) ST-T database (90 patients, $2 \mathrm{~h}$ each, 2 leads)

- Exercise ECGs (bicycle and treadmill, 1416 patients, approx. 15 min each, 12 standard leads)

Reference annotations for visible TWA episodes were created by first analyzing the data with an overly sensitive threshold and then manually verifying and correcting the first pass episodes. 


\subsection{The TWA algorithm}

First the baseline shift of the current PQRST complex of the incoming ECG is corrected by subtraction of a cubic spline. The cubic spline is calculated on the basis of three points taken from the isoelectric line preceding three consecutive QRS complexes. The cubic spline algorithm has the advantage of removing baseline artifact without affecting the low frequency content of the ECG signal, and therefore has no negative effect on the $\mathrm{T}$-wave alternans measurement.

In the next step the ECG is tested for noisy beats. Noisy beats are detected by analyzing the high and middle-frequency content in the ST-T segment. Noisy beats are excluded from further processing. Beats excluded are always excluded in pairs (e.g. noisy beat and an adjacent beat) to preserve the even-odd-sequence.

Subsequently the algorithm separates odd and even beats and updates the odd median or the even median beat (see figure 2). The odd and the even median beat are updated by either a fixed increment, or a fraction of the difference between the median template and the incoming beat, whichever is less. The default update fraction is $1 / 32$, but other factors are selectable, namely $1 / 8,1 / 16$ or 1/64. These factors will affect the rate at which the median template tracks changes in the incoming signal. A smaller factor (i.e. 1/64) will be slower in tracking, but will be more resilient to noise.

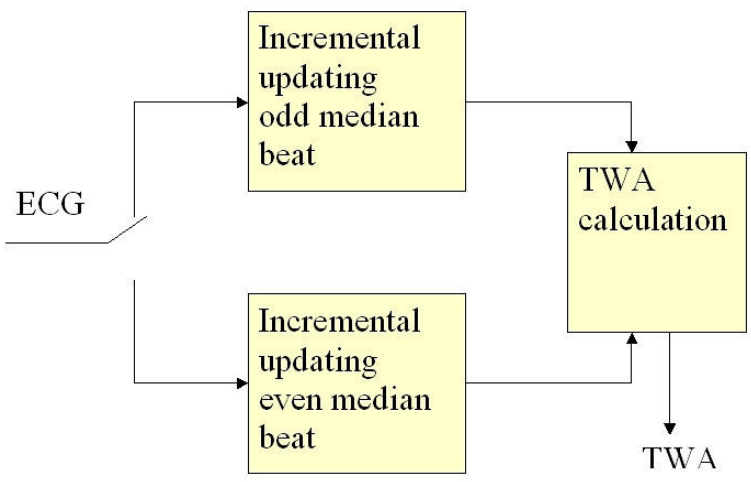

Figure 2: Block diagram of the core TWA algorithm

Then both the odd and even median beats are aligned to a cubic spline (see: 2.2.1 cubic alignment). This method has a tremendous effect in reducing baseline artifact within the PQRST cycle, without distorting the TWA measurement.

The TWA value is derived by calculating the maximal difference between odd and even beats in the range from QRS offset to T-wave offset. A nonlinear filter is applied to compute the maximal difference (see: 2.2.2 nonlinear filter for TWA calculation). The amount of the maximal difference is the TWA value.
The noise value is the average of the variability of the ST-T segments in the odd beats and the variability in the even beats (see 2.2.3 intra-class variability). The noise value is used to calculate an estimate of the signal to noise ratio.

The TWA value is annotated with a question mark if the following conditions exist:

- the heart rate exceeds the high limit (125 bpm)

- the signal to noise ratio noise value is less than 3

- too many noisy beats are excluded

TWA values with a question mark are not used for calculation of a maximum TWA value, for instance, and are not included in the trend curves.

Limiting the heart rate prevents false positive TWA values, since a higher occurrence of artifacts is expected with high heart rate.

Since a premature beat can affect the T-wave of the preceding beat, ectopic beats are detected and excluded together with the preceding beat from incremental updating of odd and even median beats.

\subsubsection{Cubic alignment}

The baseline wander cannot be fully corrected with the cubic spline algorithm described above. Since only points in the isoelectric area preceding the QRS complexes are used, the baseline wander can be removed only to a limited extent. To increase the effectiveness it seems logical to take an additional point for cubic spline correction, namely a point after the T-end. But the amplitude in the isoelectric area before the QRS complex and the amplitude in the isoelectric area between $\mathrm{T}$-wave and P-wave are different. The isoelectric area before the QRS complex is influenced by the repolarization of the Pwave. Other reasons for different amplitudes in both "isoelectric areas" are short PR intervals or a merging of $\mathrm{P}$ and T-waves, for example. Applying the cubic spline algorithm to points before the QRS complex and also to points after the $\mathrm{T}$-wave will cause artificial baseline wander, and therefore incorrect T-wave alternans values.

The cubic alignment algorithm was developed to avoid these problems. It aligns both the even and the odd median beat to a target cubic spline in the following way:

1. A target cubic spline is calculated on the basis of three points located between the odd and even median beats, before the P-wave, before the QRS complex and after the T-wave.

2. A cubic spline from the odd median beat is calculated on the basis of three points located on the odd median beat curve, before the P-wave, before the QRS complex and after the T-wave.

3. A cubic spline from the even median beat is calculated in the same way as for the odd median 
beat (see above)

4. From the odd median beat the difference between odd spline and target spline is subtracted sample by sample.

5. From the even median beat the difference between even spline and target spline is subtracted sample by sample.

The algorithm is tolerant to the location of the points, even when atrial repolarization, a short PR interval or a $\mathrm{T}$-wave merged with a $\mathrm{P}$-wave hides the isoelectric line before the QRS complex or after the T-wave. The improvement can be seen in comparing figures 3 and 4 .

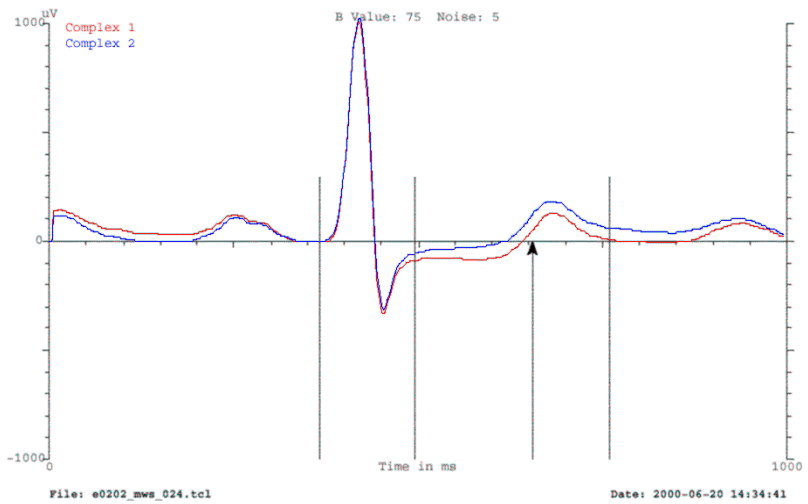

Figure 3. Example of insufficient correction of baseline wander. Superimposed odd and even median beats show a value of 75. The separation of medians outside the ST-T segment indicates the measurement is caused by baseline artifact.

\subsubsection{Nonlinear filter for TWA calculation}

When calculating the TWA value with the maximum difference between odd and even beat in the area between QRS end and T-end, high frequency noise can contaminate the TWA value. Therefore, a nonlinear filter is applied to minimize the effects of high frequency noise.

The filter uses two $20 \mathrm{~ms}$ windows, one in the odd, the other in the even median beat, both starting at QRS offset. The minimal difference between the windows is selected and stored. Then the windows are moved one step towards the end of the T-wave. Again, the minimal difference is selected and stored and the windows are moved once more. The procedure is repeated until the windows reach $\mathrm{T}$-end. Then the maximum of the stored values is taken as the TWA value.

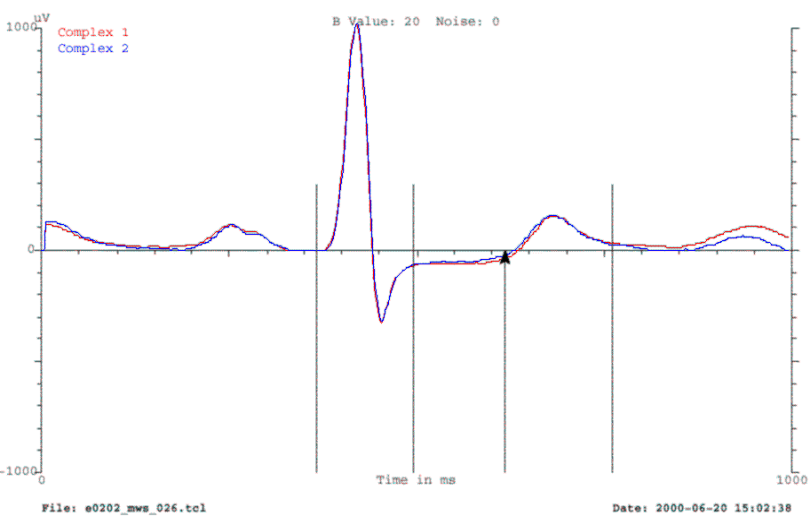

Figure 4. Same odd and even median beats as in figure 3, but with alignment to a cubic spline. The TWA value is reduced from 75 to $20 \mu \mathrm{V}$ by the cubic alignment algorithm.

\subsubsection{Intra-class variability}

The intra-class variability is simply the average of the TWA value of the odd beats and the TWA value of the even beats, as a measure of within class variability. The result is taken as the noise value and used for calculation of signal to noise ratio between the TWA value (difference between odd and even classes) and this noise value (intra-class variability).

In a clean ECG with TWA the intra-class variability in the ST-T-segments in the odd beats as well as in the even beats is small (see figure 5). Patient movement, respiration, footfalls or pedaling are likely to increase this variability. The higher the variability the less reliable is the TWA value.

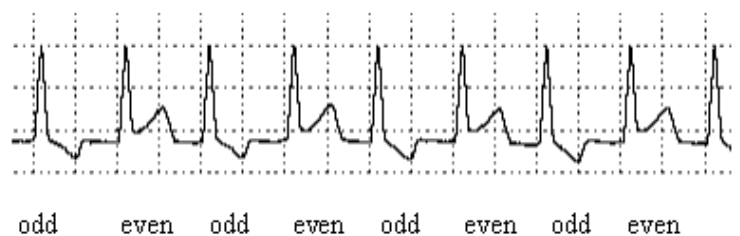

Figure 5. Significant T-wave alternans with low intraclass variability. The ST-T segments of the odd beats are different from the even beats, but all ST-T segments of odd beats have the same shape, and also all ST-T segments of all even beats have the same shape. 


\section{Results}

Measurement accuracy using simulated ECGs

- $\quad$ TWA measurement in the range from $800 \mu \mathrm{V}$ to $1 \mu \mathrm{V}$ are accurate to $1 \mu \mathrm{V}$

- the reduction of the influence of noise by a factor greater than 10 was achieved

- $\quad$ sinusoidal base line shifts of $500 \mu \mathrm{V}$ peak to peak amplitude have minimal impact on the $\mathrm{T}$-wave alternans values.

With the real ECG the TWA detection sensitivity and specificity were calculated using the following episode definitions:

- true positive: reference episode overlaps the algorithm episode

- false negative: reference episode with no overlapping algorithm episode

- true negative: every patient

- false positive: with no overlapping reference episode

\begin{tabular}{|l|c|c|}
\hline & Sensitivity & Specificity \\
\hline Exercise ECGs (1416) & $92.0 \%$ & $91.2 \%$ \\
\hline Holter ECGs (90) & $88.2 \%$ & $90.0 \%$ \\
\hline
\end{tabular}

Table 1. Visible TWA detection performance in real exercise and Holter ECGs. Sensitivity $=\mathrm{Tp} /(\mathrm{Tp}+\mathrm{Fn})$, Specificity $=\mathrm{Tn} /(\mathrm{Tn}+\mathrm{Fp}), \mathrm{Tp}=$ number of true positives, $\mathrm{Fn}=$ number of false negatives, $\mathrm{Tn}=$ number of true negatives, $\mathrm{Fp}=$ number of false positives

The threshold for the algorithm for detection of visible TWA was $30 \mu \mathrm{V}$. A limitation of this study is that only visible TWA episodes were definable, and some TWA episodes may be hidden in ECG areas with noise and artifacts. Nevertheless the way of measuring the performance was a good tool during development and for verification of the algorithm functionality in real ECG environment

\section{Discussion and conclusions}

We have significantly improved the acceptance and rejection capability for artifacts and noise of the original method by implementing the novel algorithms, cubic alignment, nonlinear filter for TWA calculation, and intra class variability, developed by using real Holter and exercise test ECGs acquired with normal electrodes. Therefore we realize that it is sufficient to acquire the ECG for TWA examinations with normal electrodes.

With slow incremental updating, e.g. with a factor of $1 / 32$, the specificity is optimized for Holter ECGs and exercise ECGs. But the sensitivity is reduced for detecting short TWA episodes. These short TWA episodes are also of great importance in predicting ventricular fibrillation [3]. For detecting short TWA episodes a factor of $1 / 8$ is more appropriate, if the ECG is clean.

Despite the improved acceptance and rejection capability for noise and artifacts, noise related false positive TWA values cannot be completely eliminated, especially when the pedaling or respiration rate comes close to half the heart rate, or a footfall occurs with every heart beat.

The T-wave alternans is calculated in the time domain. Therefore, most results can be easily verified by reviewing the odd and even median beat superimposed upon the other (see figure 6).

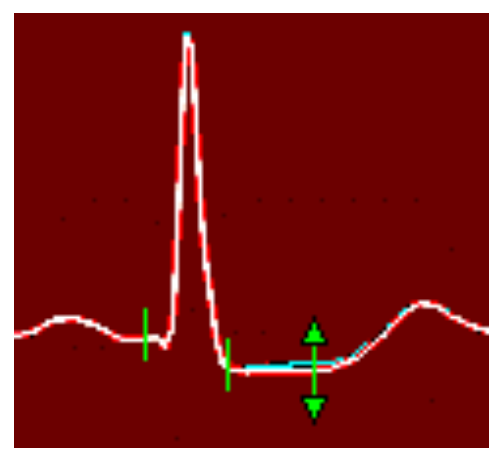

Figure 6. Odd and even median beat superimposed. Ramplitude $1.45 \mathrm{mV}$, TWA value $20 \mu \mathrm{V}$, visible in the STsegment.

\section{References}

[1] David S.Rosenbaum, Lance E.Jackson, Joseph M.Smith, Hasan Garan, Jeremy Ruskin, and Richard J.Cohen. Electrical alternans and vulnerability to ventricular arrhythmias. The New England Journal of Medicine, 330(4):235-241, January 271994.

[2] Bruce D.Nearing and Richard L.Verrier. System and method for quantifying alternation in an electrocardiogram signal. Patent No.: US 6,169,919 B1, Jan. 2, 2001

[3] Bruce D.Nearing and Richard L.Verrier. Modified moving average analysis of T-wave alternans to predict ventricular fibrillation with high accuracy. J. Appl Physiol. Vol 92, 541-549, 2002

Address for correspondence

Willi Kaiser

GE Healthcare Information Technologies

Munzinger Straße 3

D-79111 Freiburg, Germany

willi.kaiser@med.ge.com 